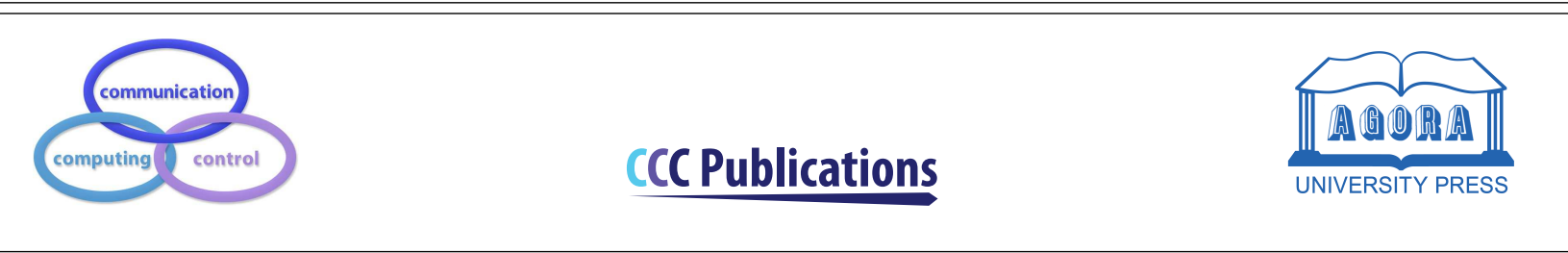

\title{
Matrix Game with Payoffs Represented by Triangular Dual Hesitant Fuzzy Numbers
}

\author{
Z. Yang, Y. Song
}

\author{
Zhihui Yang* \\ School of Science \\ East China University of Technology \\ Nanchang, 330013, China \\ *Corresponding author: zhhyang@ecut.edu.cn

\section{Yang Song} \\ Zhumadian Technician College \\ Zhumadian, 463000, China \\ hnsongyang@126.com
}

\begin{abstract}
Due to the complexity of information or the inaccuracy of decision-makers' cognition, it is difficult for experts to quantify the information accurately in the decision-making process. However, the integration of the fuzzy set and game theory provides a way to help decision makers solve the problem. This research aims to develop a methodology for solving matrix game with payoffs represented by triangular dual hesitant fuzzy numbers (TDHFNs). First, the definition of TDHFNs with their cut sets are presented. The inequality relations between two TDHFNs are also introduced. Second, the matrix game with payoffs represented by TDHFNs is investigated. Moreover, two TDHFNs programming models are transformed into two linear programming models to obtain the numerical solution of the proposed fuzzy matrix game. Furthermore, a case study is given to to illustrate the efficiency and applicability of the proposed methodology. Our results also demonstrate the advantage of the proposed concept of TDHFNs.
\end{abstract}

Keywords: matrix game, hesitant fuzzy set, triangular dual hesitant fuzzy numbers, multiobjective programming.

\section{Introduction}

Game theory, an effective method to handle the problems in which two conflicting interests exist, has been extensively applied in some fields, such as marketing and finance. In classic game theory, payoffs are assumed to be precise. However, many problems cannot be explained in a simple crisp sense due to the complexity of the problem or a lack of adequate information. Generally, such game problems can be solved by combining game theory with fuzzy set (FS)[34]. Fuzzy logic-based method is important for application on many disciplines $[22,25,28]$. Decision makers sometimes have some 
degree of hesitation in some situations. Their preference information is considered in terms of support, opposition and their confidence and nonconfidence level about the approximation. The hesitant fuzzy set (HFS) was originally introduced as an extension of the FS by Torra [26]. The membership function of HFS is a set composed of several numbers in the interval [0,1], and HFS can describe the hesitation of the players. Nevertheless, HFS cannot reflect the degree of opposition expressed by the players. Atanassov proposed an intuitionistic fuzzy set (IFS) characterized by two functions: a membership function and a non-membership function [5]. However, an IFS cannot describe the hesitation or difference in opinions of decision makers. Therefore, dual hesitant fuzzy set (DHFS) was proposed by Zhu et al. [35] to solve the above problems. DHFS consists of two components:a membership hesitancy function and a non-membership hesitancy function.DHFS can effectively describe the different confidence and nonconfidence level about attitudes (support and opposition) of experts.

After Aubin implemented fuzzy theory in a cooperative game model [6], many research works have been published on this topic. Borkotokey and Neog introduced a dynamic resource allocation mechanism within the framework of cooperative games with fuzzy coalitions [11]. Wu studied the cores and dominance cores of cooperative games with fuzzy payoffs [29]. Considering the solution concepts for fuzzy coalition games under a certain participation level, Yu et al. investigated the coincident fuzziness form for games with fuzzy coalition [33]. Yang et al. developed a methodology for solving bi-matrix games with intuitionistic fuzzy number payoffs [32]. Fei and Li proposed a bilinear programming method for solving interval bi-matrix games [13]. An and Li discussed methodologies for solving bi-matrix games with intuitionistic fuzzy information [3,4].

Two-person zero-sum games, which are often called matrix game for short, are an important type of non-cooperative game. Many research works have contributed to the research of the matrix game. Vijay et al. introduced a fuzzy relation approach to develop two-person zero-sum matrix game with fuzzy goals and fuzzy payoffs [27]. Collins and Hu considered interval-valued game matrices and extended the results of classic strictly determined matrix game to fuzzily determined interval games [12]. Gao et al. investigated two-player zero-sum games with fuzzy payoffs to define the best responses of the players[15]. They proposed three types of credibilistic equilibria and their existence theorem [15]. Nan and Li investigated methods for solving matrix game with intuitionistic fuzzy information [23,24]. $\mathrm{Li}$ developed a fast approach to compute fuzzy game values for two-person zero-sum games with triangular fuzzy number payoffs [19]. Li and Hong developed a methodology for solving constrained matrix game with trapezoidal fuzzy number payoffs [20]. Aggarwal et al. investigated a class of linear programming problems with fuzzy goals or fuzzy constraints to solve two-person zero-sum matrix game problems with IFSs [2]. Gao and Yu proposed three types of credibilistic equilibria and credibilistic subgame perfect equilibria for fuzzy extensive games [16]. Bhaumik et al. put forward the robust ranking technique to solve matrix game with triangular intuitionistic fuzzy number payoffs [9]. As deterministic payoffs are often unavailable, Figueroa-García et al. developed an uncertain basedmatrix game model involving interval-valued fuzzy payoffs [14]. Jana and Roy discussed dual hesitant fuzzy matrix game based on the similarity measure between DHFS [18]. Bhaumik and Roy proposed a new aggregation operator for solving intuitionistic interval-valued hesitant fuzzy matrix games [8]. Bhaumik et al. investigated two-person non-zero-sum game with payoff values represented by the hesitant interval-valued intuitionistic fuzzy-linguistic term set [10].Furthermore, matrix game theory has various applications, including decision making and intelligent manufacturing [30,31]. Therefore, it is necessary to research matrix game with DHFS.

In this paper, we propose the concept of triangular dual hesitant fuzzy numbers (TDHFNs), which can reflect the different level for decision-makers' attitudes (support and opposition). Moreover, the cut set of TDHFNs is also investigated. Our work is focus on developing the matrix game with payoffs represented by TDHFNs.

The remainder of this paper is organized as follows. In section 2, definitions and properties of TDHFNs are described. Section 3 proposes a programming method for matrix game with payoffs represented by TDHFNs. In section 4, an empirical case is given to illustrate the proposed method. Finally, section 5 draws conclusions. 


\section{Definition of TDHFNs and solution for matrix game with payoffs represented by TDHFNs}

In this section, the definition and some properties of TDHFNs are presented. Using the definitions and operations of TDHFNs, the ranking order of TDHFNs is described. Based on the ranking order and the operations, the solution of the matrix game can be obtained by solving a pair of linear programming model.

Considering a dual hesitant fuzzy set (DHFS) expressed as $E=\left\{\left\langle x, f_{E}(x)\right\rangle \mid x \in X\right\}[35]$, where $f_{E}(x)=<\underline{a_{t}}, a_{t}, \overline{a_{t}} ; \mu_{\widetilde{a_{t}}}, \nu_{\widetilde{a_{t}}}>(t=1, \cdots, N)$ is a dual hesitant fuzzy number representing the possible membership and non-membership degrees of element $x \in X$ to $E$, respectively.

The possible membership functions are formulated as follows

$$
\mu_{\tilde{a}_{t}}(x)=\left\{\begin{array}{c}
\frac{x-\underline{a}_{t}}{a_{t}-\underline{a}_{t}} w_{\tilde{a}_{t}}, \quad \underline{a}_{t} \leq x \leq a_{t}, \\
\frac{\overline{\bar{a}}_{t}-x}{\bar{a}_{t}-a_{t}} w_{\tilde{a}_{t}}, \quad a_{t}<x \leq \bar{a}_{t}, \\
0, \quad x<\underline{a}_{t} \quad \text { or } \quad x>\bar{a}_{t} .
\end{array}\right.
$$

The possible non-membership functions are formulated as follows

$$
v_{\tilde{a}_{t}}(x)=\left\{\begin{array}{cc}
\frac{a_{t}-x+u_{\tilde{a}_{t}}\left(x-\underline{a}_{t}\right)}{a_{t}-a_{t}}, & \underline{a}_{t} \leq x \leq a_{t}, \\
\frac{x-a_{t}+u_{\tilde{a}_{t}}\left(\bar{a}_{t}-x\right)}{\bar{a}_{t}-a_{t}}, & a_{t}<x \leq \bar{a}_{t}, \\
1, & x<\underline{a}_{t} \quad \text { or } \quad x>\bar{a}_{t} .
\end{array}\right.
$$

where $w_{\tilde{a}_{t}}$ and $u_{\tilde{a}_{t}}$ represent the maximum possible membership degree and the minimum possible non-membership degree of element $x$ belonging to $E$, respectively. Moreover, these values satisfy the conditions $0 \leq w_{\tilde{a}_{t}} \leq 1,0 \leq u_{\tilde{a}_{t}} \leq 1$, and $0 \leq w_{\tilde{a}_{t}}+u_{\tilde{a}_{t}} \leq 1$.

Definition 1. $\tilde{a}=<\left(\underline{a}_{t}, a_{t}, \bar{a}_{t}\right) ; w_{\tilde{a}_{t}}, u_{\tilde{a}_{t}}>$ is called a triangular dual hesitant fuzzy number (TDHFN).

If $\underline{a}_{t} \geq 0, \bar{a}_{t}>0(t=1, \cdots, N)$, then $\tilde{a}=<\left(\underline{a}_{t}, a_{t}, \bar{a}_{t}\right) ; w_{\tilde{a}_{t}}, u_{\tilde{a}_{t}}>$ is called a positive TDHFN, denoted by $\tilde{a}>{ }_{d} 0$. Similarly, if $\bar{a}_{t} \leq 0, \underline{a}_{t}<0(t=1, \cdots, N)$, then $\tilde{a}=<\left(\underline{a}_{t}, a_{t}, \bar{a}_{t}\right) ; w_{\tilde{a}_{t}}, u_{\tilde{a}_{t}}>$ is called a negative TDHFN, denoted by $\tilde{a}<_{d} 0$. Let $>_{d}$ and $<_{d}$ denote "dual hesitant fuzzy greater than" and "dual hesitant fuzzy less than", respectively.

Definition 2. Let $\tilde{a}$ be a TDHFN. The $\alpha$-cut set of $\tilde{a}$ is defined as $\tilde{a}_{\alpha}=\left\{x \mid \mu_{\tilde{a}_{t}}(x) \geq \alpha\right\}$. The $\beta$-cut set of $\tilde{a}$ is $\tilde{a}_{<\alpha, \beta>}=\left\{x \mid \mu_{\tilde{a}_{t}} \geq \alpha, v_{\tilde{a}_{t}} \leq \beta\right\}$, where $\alpha \in\left[0, \min \left\{w_{\tilde{a}_{t}}\right\}\right], \beta \in\left[\max \left\{u_{\tilde{a}_{t}}\right\}, 1\right]$ and $0 \leq \alpha+\beta \leq 1$.

According to definition 2, the following theorem can be obtained.

Theorem 1. Let $\tilde{a}$ be a TDHFN, $\tilde{a}_{<\alpha, \beta>}$ can be calculated as $\tilde{a}_{<\alpha, \beta>}=\tilde{a}_{\alpha} \cap \tilde{a}^{\beta}$.

Definition 3. If $\tilde{a}_{\alpha} \leq \tilde{b}_{\alpha}$ and $\tilde{a}^{\beta} \leq \tilde{b}^{\beta}$, then $\tilde{a}$ is called not greater than $\tilde{b}$, which denoted as $\tilde{a} \leq_{d} \tilde{b}$.

Similarly to the intuitionistic fuzzy number ranking relationship, let $\tilde{a}_{i}=<\left(\underline{a}_{i}, a_{i}, \bar{a}_{i}\right) ; w_{\tilde{a}_{i}}, u_{\tilde{a}_{i}}>$ and $\tilde{a}_{j}=<\left(\underline{a}_{j}, a_{j}, \bar{a}_{j}\right) ; w_{\tilde{a}_{j}}, u_{\tilde{a}_{j}}>$, if $\tilde{a}_{i \alpha} \leq \tilde{a}_{j \alpha}$ and $\tilde{a}_{i}^{\beta} \leq \tilde{a}_{j}^{\beta}$, then $\tilde{a}_{i} \leq \tilde{a}_{j}(0 \leq i, j \leq N)$.

Remark 1. In this paper, the elements of $\tilde{a}$ are arranged according to the increasing order.

Definition 4. Let $\tilde{a}=\left(\tilde{a}_{1}, \cdots, \tilde{a}_{N}\right)$, then $\xi=\varsigma \tilde{a}_{N}+(1-\varsigma) \tilde{a}_{1}(0 \leq \varsigma \leq 1)$ is called an additional element of TDHFN $\tilde{a}$. where $\varsigma$ can be decided according to the risk preference of decision maker.

Definition 5. Let $\tilde{a}=<\left(\underline{a}_{t}, a_{t}, \bar{a}_{t}\right) ; w_{\tilde{a}_{t}}, u_{\tilde{a}_{t}}>\left(t=1, \cdots, N_{1}\right)$ and $\tilde{b}=<\left(\underline{b}_{t}, b_{t}, \bar{b}_{t}\right) ; w_{\tilde{b}_{t}}, u_{\tilde{b}_{t}}>(t=$ $\left.1, \cdots, N_{2}\right)$ be two TDHFNs. If $N_{1}=N_{2}$, then the TDHFNs are called two normalized TDHFNs.

Definition 6. Let $\tilde{a}=<\left(\underline{a}_{t}, a_{t}, \bar{a}_{t}\right) ; w_{\tilde{a}_{t}}, u_{\tilde{a}_{t}}>$ and $\tilde{b}=<\left(\underline{b}_{t}, b_{t}, \bar{b}_{t}\right) ; w_{\tilde{b}_{t}}, u_{\tilde{b}_{t}}>(t=1, \cdots, N)$ be two normalized TDHFNs, and let $\lambda$ be a real number. The arithmetic operations of TDHFNs are defined as follows.

$$
\tilde{a}+\tilde{b}=<\left(\underline{a}_{t}+\underline{b}_{t}, a_{t}+b_{t}, \bar{a}_{t}+\bar{b}_{t}\right) ; \min \left\{w_{\tilde{a}_{t}}, w_{\tilde{b}_{t}}\right\}, \max \left\{u_{\tilde{a}_{t}}, u_{\tilde{b}_{t}}\right\}>
$$




$$
\begin{aligned}
& \tilde{a} \tilde{b}=\left\{\begin{array}{l}
<\left(\underline{a}_{t} \underline{b}_{t}, a_{t} b_{t}, \bar{a}_{t} \bar{b}_{t}\right) ; \min \left\{w_{\tilde{a}_{t}}, w_{\tilde{b}_{t}}\right\}, \max \left\{u_{\tilde{a}_{t}}, u_{\tilde{b}_{t}}\right\}>, \quad \tilde{a}>_{d} 0, \tilde{b}>_{d} 0, \\
<\left(\underline{a}_{t} \bar{b}_{t}, a_{t} b_{t}, \bar{a}_{t} \underline{b}_{t}\right) ; \min \left\{w_{\tilde{a}_{t}}, w_{\tilde{b}_{t}}\right\}, \max \left\{u_{\tilde{a}_{t}}, u_{\tilde{b}_{t}}\right\}>, \quad \tilde{a}>_{d} 0, \tilde{b}<_{d} 0, \\
<\left(\bar{a}_{t} \bar{b}_{t}, a_{t} b_{t}, \underline{a}_{t} \underline{b}_{t}\right) ; \min \left\{w_{\tilde{a}_{t}}, w_{\tilde{b}_{t}}\right\}, \max \left\{u_{\tilde{a}_{t}}, u_{\tilde{b}_{t}}\right\}>, \quad \tilde{a}<_{d} 0, \tilde{b}<_{d} 0 .
\end{array}\right. \\
& \lambda \tilde{a}=\left\{\begin{array}{l}
<\left(\lambda \underline{a}_{t}, \lambda a_{t}, \lambda \bar{a}_{t}\right) ; w_{\tilde{a}_{t}}, u_{\tilde{a}_{t}}>, \quad \lambda>0, \\
<\left(\lambda \bar{a}_{t}, \lambda a_{t}, \lambda \underline{a}_{t}\right) ; w_{\tilde{a}_{t}}, u_{\tilde{a}_{t}}>, \quad \lambda<0 .
\end{array}\right. \\
& \tilde{a}^{-1}=<\left(1 / \underline{a}_{t}, 1 / a_{t}, 1 / \bar{a}_{t}\right) ; w_{\tilde{a}_{t}}, u_{\tilde{a}_{t}}>
\end{aligned}
$$

Let $\hat{a}=\left[a_{L}, a_{U}\right]$ be an interval, where $a_{L}$ and $a_{R}$ are the left and right limits of the interval $\hat{a}$, respectively.

The maximization problem with an interval-valued objective is described as follows respectively. Ishibuchi and Tanaka [17] investigated the maximization problem with an interval-valued objective as $\max \{\hat{a}\}$,s.t. $\hat{a} \in \Omega_{1}$ which is equivalent to the following bi-objective programming problem

$$
\begin{aligned}
& \max \left\{a_{L}, \frac{a_{L}+a_{U}}{2}\right\} \\
& \text { s.t. } \hat{a} \in \Omega_{1}
\end{aligned}
$$

where $\Omega_{1}$ is the set of constraint conditions.

The minimization problem with an interval-valued objective is described as $\min \{\hat{a}\}$, s.t. $\hat{a} \in \Omega_{2}$ which is equivalent to the following bi-objective programming problem

$$
\begin{aligned}
& \max \left\{a_{U}, \frac{a_{L}+a_{U}}{2}\right\} \\
& \text { s.t. } \hat{a} \in \Omega_{2}
\end{aligned}
$$

where $\Omega_{2}$ is the set of constraint conditions.

In the classical matrix game, the payoff function was often expressed by the crisp number. However, there is often some hesitant fuzzy information about the payoffs. Based on the hesitant fuzzy information, we can develop the matrix game with payoffs represented by TDHFNs. Assume that $S_{1}=\left\{\alpha_{1}, \alpha_{2}, \cdots, \alpha_{m}\right\}$ and $S_{2}=\left\{\beta_{1}, \beta_{2}, \cdots, \beta_{n}\right\}$ are the pure strategies for players $p_{1}$ and $p_{2}$, respectively. Without loss of generality, the payoff matrix for player $p_{1}$ is given by $\tilde{A}=\left(\tilde{a}_{i j}\right)_{m \times n}$, where $\tilde{a}_{i j}=<\left(\underline{a}_{i j t}, a_{i j t}, \bar{a}_{i j t}\right) ; w_{\tilde{a}_{i j t}}, u_{\tilde{a}_{i j t}}>(i=1, \cdots, m ; j=1, \cdots, n ; t=1, \cdots, N)$.

The sets of mixed strategies for players $p_{1}$ and $p_{2}$ are denoted by $X$ and $Y$, respectively, where $X=\left\{\mathbf{x}=\left(x_{1}, x_{2}, \cdots, x_{m}\right) \mid \sum_{i=1}^{m} x_{i}=1, x_{i} \geq 0\right\}, Y=\left\{\mathbf{y}=\left(y_{1}, y_{2}, \cdots, y_{n}\right) \mid \sum_{j=1}^{n} y_{j}=1, y_{j} \geq 0\right\}$. If players $p_{1}$ and $p_{2}$ choose mixed strategies $\mathbf{x} \in \mathbf{X}, \mathbf{y} \in \mathbf{Y}$, respectively. According to definition 6 , the expected payoff $E(\mathbf{x}, \mathbf{y})$ can be calculated as follows

$$
\begin{aligned}
E(\mathbf{x}, \mathbf{y}) & =\mathbf{x}^{T} \tilde{A} \mathbf{y}=\sum_{i=1}^{m} \sum_{j=1}^{n} \tilde{a}_{i j} x_{i} y_{j} \\
& =<\left(\sum_{i=1}^{m} \sum_{j=1}^{n} \underline{a}_{i j t} x_{i} y_{j}, \sum_{i=1}^{m} \sum_{j=1}^{n} a_{i j t} x_{i} y_{j}, \sum_{i=1}^{m} \sum_{j=1}^{n} \bar{a}_{i j t} x_{i} y_{j}\right) ; \min \left\{w_{\tilde{a}_{i j t}}\right\}, \max \left\{u_{\tilde{a}_{i j t}}\right\}>
\end{aligned}
$$

The interests of the two players are precisely opposite. The payoff of each player is not only related to the strategy he chooses, but also depends on the strategy chosen by the opponent. Therefore, each player considers the strategy chosen by the opponent when determining the strategy that is most beneficial to himself. Assume that the players are rational. Player $p_{1}$ should choose the mixed strategy $\mathbf{x} \in \mathbf{X}$ that maximizes $E(\mathbf{x}, \mathbf{y})$, while player $p_{2}$ is interested in choosing the mixed strategy $\mathbf{y} \in \mathbf{Y}$ that minimizes $E(\mathbf{x}, \mathbf{y})$. If player $p_{1}$ chooses strategy $\mathbf{x}$, then he could realize a payoff of at least $\min _{\mathbf{y} \in Y}\left\{\mathbf{x}^{T} \tilde{A} \mathbf{y}\right\}$ However, player $p_{1}$ wants a larger payoff, so he chooses a strategy that ensures a 
payoff of no less than $\max _{\mathbf{x} \in X} \min _{\mathbf{y} \in Y}\left\{\mathbf{x}^{T} \tilde{A} \mathbf{y}\right\}$. Similarly, if player $p_{2}$ choose strategy $\mathbf{y}$, then he could realize a payoff of at most $\max _{\mathbf{x} \in X}\left\{\mathbf{x}^{T} \tilde{A} \mathbf{y}\right\}$. However, player $p_{2}$ wants a smaller payoff, so he chooses a strategy to ensure a payoff of no more than want less payoff, so he would choose the strategy make the payoff is no more than $\min _{\mathbf{y} \in Y} \max _{\mathbf{x} \in X}\left\{\mathbf{x}^{T} \tilde{A} \mathbf{y}\right\}$.

Player $p_{1}$ chooses a strategy according to the maximin principle, while player $p_{2}$ chooses his strategy according to the minimax principle. Therefore, if a pair of mixed strategies $\left(\mathbf{x}^{0}, \mathbf{y}^{0}\right)$ satisfies $\mathbf{x}^{0 T} \tilde{A} \mathbf{y}^{0}=\max _{\mathbf{x} \in X} \min _{\mathbf{y} \in Y}\left\{\mathbf{x}^{T} \tilde{A} \mathbf{y}\right\}=\min _{\mathbf{y} \in Y} \max _{\mathbf{x} \in X}\left\{\mathbf{x}^{T} \tilde{A} \mathbf{y}\right\}$, then $\mathbf{x}^{0}$ and $\mathbf{y}^{0}$ are called the optimal strategies for the two players. $\mathbf{x}^{0 T} \tilde{A} \mathbf{y}^{0}$ is the value of the mixed equilibrium situation. However, the expected payoff $E(\mathbf{x}, \mathbf{y})$ is a TDHFN with the membership degrees and non-memberships. Thus, there do not exist $\left(\mathbf{x}^{0}, \mathbf{y}^{0}\right)$ meet the two scales at the same time. In fact, the problems $\max _{\mathbf{x} \in X} \min _{\mathbf{y} \in Y}\left\{\mathbf{x}^{T} \tilde{A} \mathbf{y}\right\}$ and $\min _{\mathbf{y} \in Y} \max _{\mathbf{x} \in X}\left\{\mathbf{x}^{T} \tilde{A} \mathbf{y}\right\}$ are bi-objective programming problems.

Definition 7. Let $\tilde{\nu}$ and $\tilde{\omega}$ be TDHFNs. Assume that there exist $\left(\mathbf{x}^{*}, \mathbf{y}^{*}, \tilde{\nu}^{*}, \tilde{\omega}^{*}\right)$, where $\mathbf{x}^{*} \in X$, $\mathbf{y}^{*} \in Y$, satisfying the following conditions

(1) $\mathbf{x}^{* T} \tilde{A} \mathbf{y} \geq_{d} \tilde{\nu}^{*}$ and $\mathbf{x}^{T} \tilde{A} \mathbf{y}^{*} \leq_{d} \tilde{\omega}^{*}$,

(2) There do not exist any $\tilde{\nu} \neq \tilde{\nu}^{*}$ and $\tilde{\omega} \neq \tilde{\omega}^{*}$ such that $\tilde{\nu} \geq_{d} \tilde{\nu}^{*}$ and $\tilde{\omega} \leq_{d} \tilde{\omega}^{*}$, then, $\left(\mathbf{x}^{*}, \mathbf{y}^{*}, \tilde{\nu}^{*}, \tilde{\omega}^{*}\right)$ is the solution of the TDHFN-based matrix game, $\mathbf{x}^{*}$ and $\mathbf{y}^{*}$ are the optimal strategies of players $p_{1}$ and $p_{2}$, respectively, $\tilde{\nu}^{*}$ is the gain-floor for player $p_{1}$ and $\tilde{\omega}^{*}$ is the loss-ceiling for player $p_{2}$. Finally, $\mathbf{x}^{* T} \tilde{A} \mathbf{y}^{*}$ is the payoff of the TDHFN-based matrix game.

For the classic linear programming problem in the crisp environment, the aim is to maximize or minimize a linear objective function $[7,21]$. But in many actual situations, decision makers should specify the objective or constraint functions with fuzzy sets. Assume that each player chooses an optimal mixed strategy under the other player's pure strategy. According to definition 7, the optimal strategy $\mathbf{x}^{*}$ of player $p_{1}$ and the optimal strategy $\mathbf{y}^{*}$ of player $p_{2}$ can be achieved by solving the following pair of mathematical programming models

$$
\begin{aligned}
& \begin{array}{l}
\max \{\tilde{\nu}\} \\
\text { s.t. }\left\{\begin{array}{l}
\sum_{i=1}^{m} \tilde{a}_{i j} x_{i} \geq_{d} \tilde{\nu}(j=1,2, \cdots, n) \\
\sum_{i=1}^{m} x_{i}=1 \\
x_{i} \geq 0(i=1,2, \cdots, m)
\end{array}\right.
\end{array} \\
& \min \{\tilde{\omega}\} \\
& \text { s.t. }\left\{\begin{array}{l}
\sum_{j=1}^{n} \tilde{a}_{i j} y_{j} \leq_{d} \tilde{\omega}(i=1,2, \cdots, m) \\
\sum_{j=1}^{n} y_{j}=1 \\
y_{j} \geq 0(j=1,2, \cdots, n)
\end{array}\right.
\end{aligned}
$$

According to definition 3, model (5) can be transformed into the following programming model

$$
\begin{aligned}
& \max \left\{\begin{array}{l}
\left\{\tilde{\nu}_{\alpha}, \tilde{\nu}^{\beta}\right\} \\
\sum_{i=1}^{m}\left(\tilde{a}_{i j}\right)_{\alpha} x_{i} \geq_{d} \tilde{\nu}_{\alpha}(j=1,2, \cdots, n) \\
\sum_{i=1}^{m}\left(\tilde{a}_{i j}\right)^{\beta} x_{i} \geq_{d} \tilde{\nu}^{\beta}(j=1,2, \cdots, n) \\
\sum_{i=1}^{m} x_{i}=1 \\
x_{i} \geq 0(i=1,2, \cdots, m)
\end{array}\right.
\end{aligned}
$$


Let $\tilde{\nu}_{\alpha}=\left[\nu_{t_{\alpha}}^{L}, \nu_{t_{\alpha}}^{R}\right], \tilde{\nu}^{\beta}=\left[\nu_{t}^{\beta}, \nu_{t}^{\beta}\right],\left(\tilde{a}_{i j}\right)_{\alpha}=\left[L_{\tilde{a}_{i j t}}(\alpha), R_{\tilde{a}_{i j t}}(\alpha)\right],\left(\tilde{a}_{i j}\right)^{\beta}=\left[L_{\tilde{a}_{i j t}}(\beta), R_{\tilde{a}_{i j t}}(\beta)\right]$ be the $\alpha$-cut and $\beta$-cut of TDHFNs $\tilde{\nu}$ and $\tilde{a}_{i j}(i=1,2, \cdots, m ; j=1,2, \cdots, n)$, respectively. According to the interval-valued rank relationship and definition 4, model (7) can be transformed into the following programming model

$$
\begin{aligned}
& \max \left\{\left[\nu_{t_{\alpha}}^{L}, \nu_{t_{\alpha}}^{R}\right],\left[\nu_{t_{L}}^{\beta}, \nu_{t_{R}}^{\beta}\right]\right\} \\
& \text { s.t. }\left\{\begin{array}{l}
\sum_{i=1}^{m} L_{\tilde{a}_{i j t}}(\alpha) x_{i} \geq \nu_{t_{\alpha}}^{L}(j=1,2, \cdots, n ; t=1, \cdots, N) \\
\sum_{i=1}^{m} R_{\tilde{a}_{i j t}}(\alpha) x_{i} \geq \nu_{t}^{R}(j=1,2, \cdots, n ; t=1, \cdots, N) \\
\sum_{i=1}^{m} L_{\tilde{a}_{i j t}}(\beta) x_{i} \geq \nu_{t \beta}^{L}(j=1,2, \cdots, n ; t=1, \cdots, N) \\
\sum_{i=1}^{m} R_{\tilde{a}_{i j t}}(\beta) x_{i} \geq \nu_{t}^{R}(j=1,2, \cdots, n ; t=1, \cdots, N) \\
\sum_{i=1}^{m} x_{i}=1 \\
x_{i} \geq 0(i=1,2, \cdots, m)
\end{array}\right.
\end{aligned}
$$
follows

The above proposed model can be transformed into the bi-objective linear programming model as

$$
\begin{gathered}
\max \left\{\frac{\sum_{t=1}^{N}\left(\nu_{t_{\alpha}}^{L}+\nu_{t}^{\beta}\right)}{2 N}, \frac{\sum_{t=1}^{N}\left(\nu_{t}^{L}+\nu_{t}^{\beta}+\nu_{t_{\alpha}}^{R}+\nu_{t}^{\beta}\right)}{4 N}\right\} \\
\text { s.t. }\left\{\begin{array}{l}
\sum_{i=1}^{m} L_{\tilde{a}_{i j t}}(\alpha) x_{i} \geq \nu_{t \alpha}^{L}(j=1,2, \cdots, n ; t=1, \cdots, N) \\
\sum_{i=1}^{m} R_{\tilde{a}_{i j t}}(\alpha) x_{i} \geq \nu_{t_{\alpha}}^{R}(j=1,2, \cdots, n ; t=1, \cdots, N) \\
\sum_{i=1}^{m} L_{\tilde{a}_{i j t}}(\beta) x_{i} \geq \nu_{t}^{L}(j=1,2, \cdots, n ; t=1, \cdots, N) \\
\sum_{i=1}^{m} R_{\tilde{a}_{i j t}}(\beta) x_{i} \geq \nu_{t}^{R}(j=1,2, \cdots, n ; t=1, \cdots, N) \\
\sum_{i=1}^{m} x_{i}=1 \\
x_{i} \geq 0(i=1,2, \cdots, m)
\end{array}\right.
\end{gathered}
$$

In the context of multi-objective linear programming, it is important to note that the objectives have different level of importance [17]. In such a situation, the linear weighted average method should be applied on the multi-objective linear programming problem. Objectives with more weight should be allocated more achievement levels in comparison to others [1]. Therefore, model (9) can be transformed into the following linear programming model

$$
\begin{aligned}
& \max \left\{\lambda\left(\frac{\sum_{t=1}^{N}\left(\nu_{t_{\alpha}}^{L}+\nu_{t}^{\beta}\right)}{2 N}\right)+(1-\lambda)\left(\frac{\sum_{t=1}^{N}\left(\nu_{t_{\alpha}}^{L}+\nu_{t_{L}}^{\beta}+\nu_{t_{\alpha}}^{R}+\nu_{t}^{\beta}\right)}{4 N}\right)\right\} \\
& \text { s.t. }\left\{\begin{array}{l}
\sum_{i=1}^{m} L_{\tilde{a}_{i j t}}(\alpha) x_{i} \geq \nu_{t}^{L}(j=1,2, \cdots, n ; t=1, \cdots, N) \\
\sum_{i=1}^{m} R_{\tilde{a}_{i j t}}(\alpha) x_{i} \geq \nu_{t_{\alpha}}^{R}(j=1,2, \cdots, n ; t=1, \cdots, N) \\
\sum_{i=1}^{m} L_{\tilde{a}_{i j t}}(\beta) x_{i} \geq \nu_{t}^{L}(j=1,2, \cdots, n ; t=1, \cdots, N) \\
\sum_{i=1}^{m} R_{\tilde{a}_{i j t}}(\beta) x_{i} \geq \nu_{t_{\beta}}^{R}(j=1,2, \cdots, n ; t=1, \cdots, N) \\
\sum_{i=1}^{m} x_{i}=1 \\
x_{i} \geq 0(i=1,2, \cdots, m)
\end{array}\right.
\end{aligned}
$$

The parameter $\lambda \in[0,1]$ can be determined by the decision maker. 
According to definitions 1 and 2 , the the $\alpha$-cut and $\beta$-cut set of $\tilde{a}_{i j}=<\left(\underline{a}_{i j t}, a_{i j t}, \bar{a}_{i j t}\right) ; w_{\tilde{a}_{i j t}}, u_{\tilde{a}_{i j t}}>$ $(i=1,2, \cdots, m ; j=1,2, \cdots, n ; t=1, \cdots, N)$ are represented as follows

$$
\begin{gathered}
\left(\tilde{a}_{i j t}\right)_{\alpha}=\left[\frac{\left(w_{\tilde{a}_{i j t}}-\alpha\right) \underline{a}_{i j t}+\alpha a_{i j t}}{w_{\tilde{a}_{i j t}}}, \frac{\left(w_{\tilde{a}_{i j t}}-\alpha\right) \bar{a}_{i j t}+\alpha a_{i j t}}{w_{\tilde{a}_{i j t}}}\right] \\
\left(\tilde{a}_{i j t}\right)^{\beta}=\left[\frac{(1-\beta) a_{i j t}+\left(\beta-u_{\tilde{a}_{i j t}}\right) \underline{a}_{i j t}}{1-u_{\tilde{a}_{i j t}}}, \frac{(1-\beta) a_{i j t}+\left(\beta-u_{\tilde{a}_{i j t}}\right) \bar{a}_{i j t}}{1-u_{\tilde{a}_{i j t}}}\right]
\end{gathered}
$$

Therefore, model (10) can be transformed into the following model

$$
\begin{gathered}
\max \left\{\lambda\left(\frac{\sum_{t=1}^{N}\left(\nu_{t_{\alpha}}^{L}+\nu_{t}^{\beta}\right)}{2 N}\right)+(1-\lambda)\left(\frac{\sum_{t=1}^{N}\left(\nu_{t_{\alpha}}^{L}+\nu_{t}^{\beta}+\nu_{t_{\alpha}}^{R}+\nu_{t}^{\beta}\right)}{4 N}\right)\right\} \\
\text { s.t. }\left\{\begin{array}{l}
\sum_{i=1}^{m} \frac{\left(w_{\tilde{a}_{i j t}}-\alpha\right) \underline{a}_{i j t}+\alpha a_{i j t}}{w_{\tilde{a}_{i j}}} x_{i} \geq \nu_{t \alpha}^{L}(j=1,2, \cdots, n ; t=1, \cdots, N) \\
\sum_{i=1}^{m} \frac{\left(w_{\tilde{a}_{i j t}}-\alpha\right) \bar{a}_{i j t}+\alpha a_{i j t}}{w_{\tilde{a}_{i j t}}} x_{i} \geq \nu_{t}^{R}(j=1,2, \cdots, n ; t=1, \cdots, N) \\
\sum_{i=1}^{m} \frac{(1-\beta) a_{i j t}+\left(\beta-u_{\tilde{a}_{i j t}}\right) \underline{a}_{i j t}}{1-u_{\tilde{a}_{i j t}}} x_{i} \geq \nu_{t}^{L}(j=1,2, \cdots, n ; t=1, \cdots, N) \\
\sum_{i=1}^{m} \frac{(1-\beta) a_{i j t}+\left(\beta-u_{\tilde{a}_{i j t}}\right) \bar{a}_{i j t}}{1-u_{\tilde{a}_{i j t}}} x_{i} \geq \nu_{t \beta}^{R}(j=1,2, \cdots, n ; t=1, \cdots, N) \\
\sum_{i=1}^{m} x_{i}=1 \\
x_{i} \geq 0(i=1,2, \cdots, m)
\end{array}\right.
\end{gathered}
$$

where $\nu_{t \alpha}^{L}, \nu_{t}^{\beta}, \nu_{t \alpha}^{R}, \nu_{t}^{\beta}$ and $x_{i}(i=1,2, \cdots, m)$ are decision variables, $\lambda \in[0,1], \alpha \in\left[0, \min \left\{w_{\tilde{a}_{i j t}}\right]\right.$ and $\beta \in\left[\max \left\{u_{\tilde{a}_{i j t}}, 1\right](0 \leq \alpha+\beta \leq 1)\right.$ are parameters that can be determined according to the characteristics of the actual decision problem.

For any given parameters $\lambda, \alpha$ and $\beta$, the optimal solution $\left(\mathbf{x}^{*}(\alpha, \beta), \nu_{\alpha}^{L *}, \nu_{\alpha}^{R *}, \nu_{L}^{\beta *}, \nu_{R}^{\beta *}\right)$ can be obtained by solving model (13). $\left(\mathbf{x}^{*}(\alpha, \beta), \nu_{\alpha}^{L *}, \nu_{\alpha}^{R *}, \nu_{L}^{\beta *}, \nu_{R}^{\beta *}\right)$ is the optimal solution of model (10). $\mathbf{x}^{*}(\alpha, \beta)$ is the maximin strategy of player $p_{1}$ at confidence level $(\alpha, \beta)$. The $<\alpha, \beta>$-cut set of the minimum gain $\tilde{\nu}^{*}$ of player $p_{1}$, which is represented by $\tilde{\nu}_{<\alpha, \beta>}^{*}$, can be obtained according to theorem 1 . $\tilde{\nu}_{<\alpha, \beta>}^{*}$ denotes the possible range of $\tilde{\nu}^{*}$ in the $<\alpha, \beta>$ confidence level. Specifically, if $\alpha=0, \beta=1$, it means the largest scope of the minimum gain $\tilde{\nu}^{*}$ of player $p_{1}$. If $\alpha=\min \left\{w_{\tilde{a}_{i j t}}\right\}, \beta=\max \left\{u_{\tilde{a}_{i j t}}\right\}$, then $\tilde{\nu}_{<\alpha, \beta>}^{*}$ denotes the minimum range of $\tilde{\nu}^{*}$ of player $p_{1}$.

Let $\tilde{\omega}_{\alpha}=\left[\omega_{t}^{L}, \omega_{t_{\alpha}}^{R}\right]$, and $\tilde{\omega}_{\beta}=\left[\omega_{t_{L}}^{\beta}, \omega_{t_{R}}^{\beta}\right]$ are the $\alpha$-cut set and $\beta$-cut set of TDHFN $\tilde{\omega}$, respectively. Therefore, model (6) can be transformed into the following model

$$
\begin{gathered}
\min \left\{\lambda\left(\frac{\sum_{t=1}^{N}\left(\omega_{t_{\alpha}}^{L}+\omega_{t}^{\beta}\right)}{2 N}\right)+(1-\lambda)\left(\frac{\sum_{t=1}^{N}\left(\omega_{t_{\alpha}}^{L}+\omega_{t}^{\beta}+\omega_{t_{\alpha}}^{R}+\omega_{t R}^{\beta}\right)}{4 N}\right)\right\} \\
\text { s.t. }\left\{\begin{array}{l}
\sum_{j=1}^{n} \frac{\left(w_{\tilde{a}_{i j t}}^{\beta}-\alpha\right)_{i j t}+\alpha a_{i j t}}{w_{\tilde{a}_{i j}}} y_{j} \leq \omega_{t_{\alpha}}^{L}(i=1,2, \cdots, m ; t=1, \cdots, N) \\
\sum_{j=1}^{n} \frac{\left(w_{\tilde{a}_{i j t}}-\alpha\right) \bar{a}_{i j t}+\alpha a_{i j t}}{w_{\tilde{a}_{i j t}}} y_{j} \leq \omega_{t_{\alpha}}^{R}(i=1,2, \cdots, m ; t=1, \cdots, N) \\
\sum_{j=1}^{n} \frac{(1-\beta) a_{i j t}+\left(\beta-u_{\tilde{a}_{i j t}}\right)_{i j t}}{1-u_{\tilde{a}_{i j t}}} y_{j} \leq \omega_{t \beta}^{L}(i=1,2, \cdots, m ; t=1, \cdots, N) \\
\sum_{j=1}^{n} \frac{(1-\beta) a_{i j t}+\left(\beta-u_{\tilde{a}_{i j t}}\right) \bar{a}_{i j t}}{1-u_{\tilde{a}_{i j t}}} y_{j} \leq \omega_{t \beta}^{R}(i=1,2, \cdots, m ; t=1, \cdots, N) \\
\sum_{j=1}^{n} y_{j}=1 \\
y_{j} \geq 0(j=1,2, \cdots, n)
\end{array}\right.
\end{gathered}
$$

where $\omega_{t \alpha}^{L}, \omega_{t}^{\beta}, \omega_{t \alpha}^{R}, \omega_{t R}^{\beta}$ and $y_{j}(j=1,2, \cdots, n)$ are decision variables; $\lambda \in[0,1], \alpha \in\left[0, \min \left\{w_{\tilde{a}_{i j t}}\right\}\right]$ and $\beta \in\left[\max \left\{u_{\tilde{a}_{i j t}}\right\}, 1\right], 0 \leq \alpha+\beta \leq 1$. 
For any given parameters $\lambda, \alpha$ and $\beta$, the optimal solution $\left(\mathbf{y}^{*}(\alpha, \beta), \omega_{\alpha}^{L *}, \omega_{\alpha}^{R *}, \omega_{L}^{\beta *}, \omega_{R}^{\beta *}\right)$ can be obtained by solving model (14). Similarly, $\left(\mathbf{y}^{*}(\alpha, \beta), \omega_{\alpha}^{L *}, \omega_{\alpha}^{R *}, \omega_{L}^{\beta *}, \omega_{R}^{\beta *}\right)$ is the optimal solution of model $(9) . \mathbf{y}^{*}(\alpha, \beta)$ is the minimax strategy of player $p_{2}$ at confidence level $(\alpha, \beta), \tilde{\omega}_{<\alpha, \beta>}^{*}$ is the $<\alpha, \beta>$-cut set of $\tilde{\omega}^{*}$, and $\tilde{\omega}_{<\alpha, \beta>}^{*}$ denotes the possible range of the maximum loss $\tilde{\omega}^{*}$ of player $p_{2}$ in the $\langle\alpha, \beta\rangle$ confidence level.

\section{Illustrative case}

To illustrate the proposed model, we present an illustrative example considering a monopoly company which plans to produce products with $C_{1}$ and $C_{2}$, where $C_{1}$ and $C_{2}$ are mutually replaceable products. In the current highly competitive business environment, it is very important for the enterprise to capture the customer effectively. There are two types of customer group, $A_{1}$ and $A_{2}$, in the market. As the demand for the product is basically fixed, an increase in the sales volume of one will inevitably lead to a decrease in the sales volume of the other. The company and the customer group can be regarded as two players, and the price selection for the company and the customer group can be viewed as a matrix strategy. The payoff matrix of the company is expressed as follows

$$
\tilde{A}=\left[\begin{array}{cll}
\{<(150,155,160) ; 0.8,0.1>,<(150,160,170) ; 0.9,0.1>\} & \{<(135,138,140) ; 0.7,0.2>,<(135,140,145) ; 0.8,0.1>\} \\
\{<(75,80,85) ; 0.6,0.2>,<(80,85,90) ; 0.7,0.1>\} & \{<(145,150,155) ; 0.6,0.2>,<(150,155,160) ; 0.7,0.1>\}
\end{array}\right]
$$

where $<(150,155,160) ; 0.8,0.1>$ means that the most possible price of $C_{1}$ is 155 , the lowest possible price is 150 , the highest possible price is 160 , the maximum satisfaction of $A_{1}$ is 0.8 , and the minimum dissatisfaction is 0.1 . The other values can be interpreted similarly.

For the given $\lambda=0.5$ (the other values are calculated similarly) and select $\langle\alpha, \beta\rangle$, where $\alpha \in[0,0.6], \beta \in[0.2,1]$, models (13) and (14) can be solved using Lingo11, and the optimal strategy $\mathbf{x}^{* T}(\alpha, \beta)$ of $p_{1}$ and the optimal strategy $\mathbf{y}^{* T}(\alpha, \beta)$ of $p_{2}$ can be obtained. The $\langle\alpha, \beta>$-cut set of the minimum gain of player $p_{1}$ and the maximum loss of player $p_{2}$ can be calculated according to theorem 1. The results are shown in table 1 and table 2 .

Table 1 The max-min strategy and gain-floor cut sets of player $p_{1}$

\begin{tabular}{|c|c|c|}
\hline$<\alpha, \beta>$ & $\mathbf{x}^{* T}(\alpha, \beta)$ & $\tilde{\nu}_{<\alpha, \beta>}^{*}$ \\
\hline$<0,1>$ & $(0.8235,0.1765)$ & $\{[136.76,142.65],[137.65,147.65]\}$ \\
\hline$<0.1,0.8>$ & $(0.8216,0.1784)$ & $\{[137.62,142.04],[138.79,146.56]\}$ \\
\hline$<0.2,0.7>$ & $(0.8197,0.1803)$ & $\{[138.06,141.75],[139.37,146.06]\}$ \\
\hline$<0.3,0.6>$ & $(0.8179,0.1821)$ & $\{[138.50,141.45],[139.95,145.51]\}$ \\
\hline$<0.4,0.5>$ & $(0.8160,0.1840)$ & $\{[138.95,141.17],[140.54,144.98]\}$ \\
\hline$<0.5,0.3>$ & $(0.8141,0.1859)$ & $\{[139.81,140.55],[141.68,143.90]\}$ \\
\hline$<0.6,0.2>$ & $(0.8123,0.1877)$ & $\{140.25,[142.26,143.37]\}$ \\
\hline
\end{tabular}

Table 2 The min-max strategy and loss-ceiling cut sets of player $p_{2}$

\begin{tabular}{|c|c|c|}
\hline$<\alpha, \beta>$ & $\mathbf{y}^{* T}(\alpha, \beta)$ & $\tilde{\omega}_{<\alpha, \beta>}^{*}$ \\
\hline$<0,1>$ & $(0.1765,0.8235)$ & $\{[137.65,143.53],[137.65,149.41]\}$ \\
\hline$<0.1,0.8>$ & $(0.1742,0.8258)$ & $\{[138.43,142.88],[138.92,148.05]\}$ \\
\hline$<0.2,0.7>$ & $(0.1731,0.8269)$ & $\{[138.81,142.55],[139.55,147.37]\}$ \\
\hline$<0.3,0.6>$ & $(0.1720,0.8280)$ & $\{[139.20,142.23],[140.18,146.70]\}$ \\
\hline$<0.4,0.5>$ & $(0.1709,0.8291)$ & $\{[139.59,141.91],[141.90,146.02]\}$ \\
\hline$<0.5,0.3>$ & $(0.1688,0.8312)$ & $\{[140.37,141.26],[142.08,144.67]\}$ \\
\hline$<0.6,0.2>$ & $(0.1677,0.8323)$ & $\{[140.76,140.94],[142.71,144.00]\}$ \\
\hline
\end{tabular}

According to table 1 and table 2 , if $\langle\alpha, \beta\rangle=<0,1>$, then

$$
\tilde{\nu}_{<\alpha, \beta>}^{*}=\{[136.76,142.65],[137.65,147.65]\}
$$

which denotes the greatest possible range of minimum gain $\tilde{\nu}^{*}$ of player $p_{1}$, and

$$
\tilde{\omega}_{<\alpha, \beta>}^{*}=\{[137.65,143.53],[137.65,149.41]\}
$$

denotes the greatest possible range of maximum loss $\tilde{\omega}^{*}$ of player $p_{2}$. When $\alpha$ is larger and $\beta$ is smaller, the lengths of $\tilde{\nu}_{<\alpha, \beta>}^{*}$ and $\tilde{\omega}_{<\alpha, \beta>}^{*}$ are smaller . 
From the above discussion, the concept of TDHFNs proposed in the paper has the advantage that it can take into account the uncertainty and hesitation inherent in the assessment of decision makers, and it can also simulate the psychological behavior of decision makers. The proposed matrix games with payoff represented by TDHFNs makes full use of information from both the market and consumers.

\section{Conclusion}

In this paper, a new integrated concept of the triangular fuzzy number and dual hesitant fuzzy set enables decision makers to express psychological behaviour. The matrix game with payoffs represented by TDHFNs is investigated. Based on the cut set and operational rules of TDHFNs, the matrix game with payoffs represented by TDHFNs is transformed into a multi-objective linear programming problem. Therefore, the numerical solution of the matrix game with payoffs represented by TDHFNs can derive from a pair of linear programming models. The proposed method is illustrated by the case of a market pricing problem. The approach is especially appropriate for the decision maker under hesitant fuzzy environment. More effective methods for developing matrix game theory with TDHFNs will be investigated in the future.

\section{Funding}

This work was supported in part by the National Natural Science Foundation of China (grant No. $71762001)$.

\section{Conflict of interest}

The authors declare no conflict of interest.

\section{References}

[1] Afzali, A.; Rafsanjani, M. K.; Saeid, A. B. (2016). A fuzzy multi-objective linear programming model based on interval-valued intuitionistic fuzzy sets for supplier selection, International Journal of Fuzzy Systems, 18(5), 864-874, 2016.

[2] Aggarwal, A.; Mehra, A.; Chandra, S. (2012). Application of linear programming with i-fuzzy sets to matrix games with i-fuzzy goals, Fuzzy Optimization and DecisionMaking, 11(4), 465-480, 2012 .

[3] An, J. J.; Li, D. F. (2019). A linear programming approach to solve constrained bi-matrix games with intuitionistic fuzzy payoffs, International Journal of Fuzzy Systems, 21(3), 908-915, 2019.

[4] An, J. J.; Li, D. F.; Nan, J. X. (2017). A mean-area ranking based non-linear programming approach to solve intuitionistic fuzzy bi-matrix games, Journal of Intelligent Fuzzy Systems, 33(1), 563-573, 2017.

[5] Atanassov, K. T. (1999). Intuitionistic fuzzy sets, Springer, New York, USA, 1999.

[6] Aubin, J. P. (1981). Cooperative fuzzy games, Mathematics of Operations Research, 6(1), 1-13, 1981.

[7] Bector, C. R.; Chandra, S. (2005). Fuzzy mathematical programming and fuzzy matrix games, Germany, Berlin, Springer-Verlag.

[8] Bhaumik A.; Roy S. K. (2019). Intuitionistic interval-valued hesitant fuzzy matrix games with a new aggregation operator for solving management problem, Granular Computing, 2019.

[9] Bhaumik, A.; Roy, S.K.; Li, D.F. (2017). Analysis of triangular intuitionistic fuzzy matrix games using robust ranking, Journal of Intelligent Fuzzy Systems, 33(1), 327-336, 2017. 
[10] Bhaumik, A.; Roy, S.K.; Weber, G.W. (2020). Hesitant interval-valued intuitionistic fuzzylinguistic term set approach in Prisoners' dilemma game theory using TOPSIS: a case study on Human-trafficking, Central European Journal of Operations Research, 28, 797-816, 2020.

[11] Borkotokey, S.; Neog, R. (2014). Dynamic resource allocation in fuzzy coalitions: a game theoretic model, Fuzzy Optimization and Decision Making, 13(2), 211-230, 2014.

[12] Collins, W. D.; Hu, C. (2008). Studying interval valued matrix games with fuzzy logic, Soft Computing, 12(2), 147-155, 2008.

[13] Fei, W.; Li, D.F. (2016). Bilinear programming approach to solve interval bimatrix games in tourism planning management, International Journal of Fuzzy Systems, 18(3), 504-510, 2016.

[14] Figueroa-García, J. C.; Mehra, A.; Chandra, S. (2019). Optimal solutions for group matrix games involving interval-valued fuzzy numbers, Fuzzy Sets and Systems, 362(1), 55-70, 2019.

[15] Gao, J.; Liu, Z.Q.; Shen, P. (2009). On characterization of credibilistic equilibria of fuzzy-payoff two-player zero-sum game, Soft Computing, 13(1), 127-132, 2009.

[16] Gao, J.; Yu Y. (2013). Credibilistic extensive game with fuzzy payoffs, Soft Computing, 17(4), 557-567, 2013.

[17] Ishibuchi, H.; Tanaka, H. (1990). Multiobjective programming in optimization of the interval objective function, European Journal of Operational Research, 48(2), 219-225, 1990.

[18] Jana, J.; Roy, S. K. (2019). Dual hesitant fuzzy matrix games: based on new similarity measure, Soft Computing, 23(18), 8873-8886, 2019.

[19] Li, D. F. (2012). A fast approach to compute fuzzy values of matrix games with payoffs of triangular fuzzy numbers, European Journal of Operational Research, 223(2), 421-429, 2012.

[20] Li, D. F.; Hong, F. X. (2013). Alfa-cut based linear programming methodology for constrained matrix games with payoffs of trapezoidal fuzzy numbers, Fuzzy Optimization and Decision Making, 12(2), 191-213, 2013.

[21] Liu, F.; Qu, Y.; Wang,Y. (2012). A linear programming approximation for network capacity control problem with customer choice, Journal of System and Management Sciences, 2(3), 1-8, 2012 .

[22] Mousavi, M.; Yap, H. J.; Musa, S. N.; Dawal, S. Z. M.(2017). A fuzzy hybrid GA-PSO algorithm for multi-objective AGV scheduling in FMS, International Journal of Simulation Modelling, 16(1), 58-71, 2017.

[23] Nan, J. X.; Li, D. F. (2013). Linear programming approach to matrix games with intuitionistic fuzzy goals, International Journal of Computational Intelligence Systems, 6 (1), 186-197, 2013.

[24] Nan, J. X.; Li, D. F.; Zhang, M. J. (2010). A lexicographic method for matrix games with payoffs of triangular intuitionistic fuzzy numbers, International Journal of Computational Intelligence Systems, 3(3), 280-289, 2010.

[25] Quang, H. N.; Huu, T. D. (2017). A fuzzy ANP model for assessing the construction risk of a public construction project in Vietnam, Journal of System and Management Sciences, 7(3), 79-96, 2017.

[26] Torra, V. (2010). Hesitant fuzzy sets, International Journal of Intelligent Systems, 25(6), 529-539, 2010.

[27] Vijay, V.; Mehra, A.; Chandra, S.; Bector, C. (2007). Fuzzy matrix games via a fuzzy relation approach, Fuzzy Optimization and DecisionMaking, 6(4), 299-314, 2007. 
[28] Wang, J. F.; Fei, Z. C.; Chang, Q.; Fu, Y.; Li, S. Q. (2019). Energy-saving operation of multistage stochastic manufacturing systems based on fuzzy logic, International Journal of Simulation Modelling, 18(1), 138-149, 2019.

[29] Wu, H. C. (2019). Cores and dominance cores of cooperative games endowed with fuzzy payoffs, Fuzzy Optimization and DecisionMaking, 18(2), 219-257, 2019.

[30] Xu, W.; Liu, L.; Zhang, Q.; Liu, P. (2018). Location decision-making of equipment manufacturing enterprise under dual channel purchase and sale mode, Complexity, 2018.

[31] Xu, W.; Yin, Y. (2018). Functional objectives decision-making of discrete manufacturing system based on integrated ant colony optimization and particle swarm optimization approach, Advances in Production Engineering Management, 13(4), 389-404, 2018.

[32] Yang, J.; Fei, W.; Li, D. F. (2016). Non-linear programming approach to solve bimatrix games with payoffs represented by i-fuzzy numbers, International Journal of Fuzzy Systems, 18(3), 492$503,2016$.

[33] Yu, X.; Zhang, Q.; Zhou, Z. (2018). Linear fuzzy game with coalition interaction and its coincident solutions, Fuzzy Sets and Systems, 349, 1-22, 2018.

[34] Zadeh, L. A. (1965). Fuzzy sets, Information and Control, 8(3), 338-353, 1965.

[35] Zhu, B.; Xu, Z.; Xia, M. (2012). Dual hesitant fuzzy sets, Journal of Applied Mathematics, 13 pages, 2012.

\section{(c) (i) (9)}

Copyright (C)2020 by the authors. Licensee Agora University, Oradea, Romania.

This is an open access article distributed under the terms and conditions of the Creative Commons Attribution-NonCommercial 4.0 International License.

Journal's webpage: http://univagora.ro/jour/index.php/ijccc/

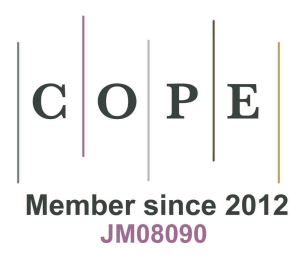

This journal is a member of, and subscribes to the principles of, the Committee on Publication Ethics (COPE).

https://publicationethics.org/members/international-journal-computers-communications-and-control

Cite this paper as:

Yang, Z.; Song, Y. (2020). Matrix Game with Payoffs Represented by Triangular Dual Hesitant Fuzzy Numbers, International Journal of Computers Communications \& Control, 15(3), 3854, 2020. https://doi.org/10.15837/ijccc.2020.3.3854 